\title{
Cardioprotective properties of a novel enriched yogurt with inhibitors of Platelet Activating Factor (PAF)
}

\author{
Maria Detopoulou, Elizabeth Fragopoulou, Anastasia Mikellidi, Ioanna Vlachogianni, \\ Marianna Xanthopoulou, Chrysa Argyrou, Tzortzis Nomikos, Mary Yannakoulia and \\ Smaragdi Antonopoulou \\ Department Nutrition and Dietetics, School of Health Science and Education, Harokopio University, Athens, Greece
}

\section{Abstract}

Platelet Activating Factor (PAF) is a pro-inflammatory mediator of atherogenesis. Food enriched with PAF inhibitors may have potential cardioprotective properties. In this attempt our group along with a well-known Greek dairy company incorporated bioactive ingredients (BAL) from olive-oil by-products, rich in PAF receptor specific inhibitors, into another typical food of the Mediterranean diet, namely yogurt. The aim of the present study was to evaluate the cardioprotective properties of the enriched yogurt in apparently healthy volunteers.

Ninety two apparently healthy volunteers (35-65 years) were randomly allocated into three groups by block-randomization based on age, sex and BMI. The volunteers in Group A did not consume any yogurt. The volunteers of Group B consumed a non-enriched yogurt daily. The Group $\mathrm{C}$ volunteers ate daily an enriched yogurt. The intervention lasted 8 weeks. Volunteers' adherence to the study protocol was assessed by two $24 \mathrm{~h}$ recalls every two weeks conducted by trained personnel. Blood and urine samples were collected at baseline, at 4th week and at 8 th week of the intervention and biochemical and inflammatory markers were determined. In addition, platelet aggregation in volunteers' platelet rich plasma against PAF, ADP and TRAP were measured and the results expressed as the concentration of the agonist that cause $50 \%$ of the maximum aggregation $\left(\mathrm{EC}_{50}\right.$, efficient concentration fifty values).

Among classical anthropometric and biochemical markers, only a decrease of blood pressure (systolic and diastolic) levels was observed $\left(\mathrm{p}_{0-8 \text { weeks }}<0.05\right)$ in group B but with no clinical significance. Reduced levels of IL-10 was observed in group C compared to $\mathrm{B}$ both in the meantime $\left(\mathrm{p}_{4 \text { weeks }}=0.01\right)$ and at the end of the intervention $\left(\mathrm{p}_{8 \text { weeks }}=0.04\right)$. Furthermore, platelet sensitivity against PAF was only decreased (higher $\mathrm{EC}_{50} \mathrm{PAF}$ ) in group $\mathrm{C}\left(\mathrm{p}_{0-4 \text { weeks }}=0.005\right.$ and $\left.\mathrm{p}_{0-8 \text { weeks }}=0.05\right)$. A trial effect was observed in the meantime among the three groups $(\mathrm{p}=0.03)$.

The consumption of the enriched yogurt led to attenuation of subclinical inflammation and platelet sensitivity to thrombotic stimuli in apparently healthy volunteers, indicating a potential cardioprotective role of PAF inhibitors.

The work is funded by SYNERGASIA 2011 PROGRAMME. This Programme is co-funded by the European Regional Development Fund and National Resources, Project Code 11SYN-2-652.

\section{Conflict of Interest}

There is no conflict of interest 